
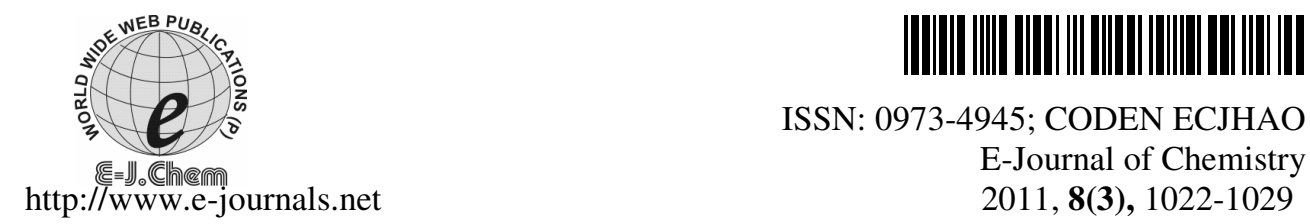

ISSN: 0973-4945; CODEN ECJHAO

E-Journal of Chemistry 2011, 8(3), 1022-1029

\title{
Synthesis and Antimicrobial Studies of Some New 3-Isoxazoline Substituted Phthalazine Methylsulfonyl Oxadiazoles
}

\author{
A.M. SRIDHARA ${ }^{\S}$, K. R. VENUGOPALA REDDY*, J. KESHAVAYYA ${ }^{\mathbb{I}}$, \\ D.S. AMBIKA ${ }^{\#}$, SANATHKUMAR GOUD ${ }^{\S}$ and S.K. PEETHAMBAR
}

\author{
${ }^{\S}$ Advinus Therapeutics Ltd, 21 \& 22 \\ Peenya II Phase, Bangalore-560058, India \\ *Department of Studies in Industrial Chemistry \\ Kuvempu University, Shankaragatta -577 451, Karnataka, India \\ II Department of studies in Chemistry \\ Kuvempu University, Shankaragatta-577 451, Karnataka, India \\ "Dept. of Plant Pathology, \\ G.K.V.K, UAS, Bangalore-65, India \\ Department of Biochemistry, Kuvempu University \\ Shankaragatta-577 451, Karnataka, India \\ venurashmi30@rediffmail.com
}

Received 25 August 2010; Revised 19 November 2010; Accepted 15 December 2010

\begin{abstract}
A series of new 3-isoxazoline substituted phthalazine methylsulfonyloxadiazoles were prepared from methyl 2-(4-oxo-3,4-dihydrophthalazin-1-yl) acetate. The structure of synthesized compounds were characterized by spectral data and screened for their antimicrobial activities against various bacteria and fungi strains. Several of these compounds showed antimicrobial activity.
\end{abstract}

Keywords: Phthalazin-1(2H)-one, 1,3,4-Oxadiazole, Isoxazoline, Phthalazine acetic acid

\section{Introduction}

Phthalazin-1(2H)-ones, methylsulfanyl oxadiazoles and isoxazolines serves as some important building blocks for biological active molecules. Figure 1 has been revealed by these three important biological active pharmacophore component systems. 


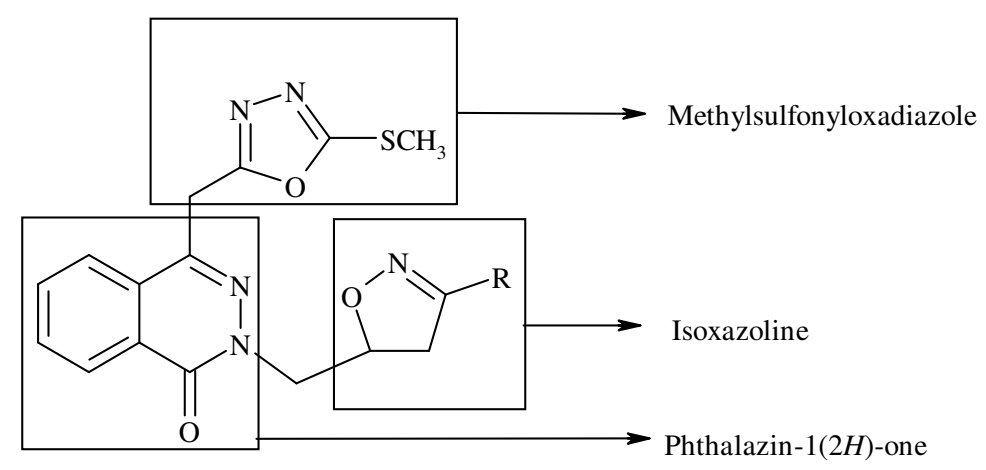

Figure 1. Phthalazine isoxazolines scaffold

Phthalazin-1(2H)-one derivatives are of considerable interest due to their antidiabatic ${ }^{1}$ antiallergic ${ }^{2}$, antiasthmatic ${ }^{3}$, antihypertensive ${ }^{4}$, vasorelaxant ${ }^{5}$, aldose reductase inhibitor ${ }^{6}$, and antimicrobial ${ }^{7}$ activities. Moreover a number of established drug molecules like hydralazine ${ }^{8}$, budralazine ${ }^{9}$, azelastine ${ }^{10}$, ponalrestat $^{11}$ and zopolrestat ${ }^{12}$ are accessible starting from the corresponding phthalazinones. The second pharmacophore component is isoxazoline, which represents an important class of heterocyclic compounds with broad spectrum of biological activities. Substituted isoxazolines have revealed anti-influenza virus $^{13}$, antifungal ${ }^{14}$, antitubercular ${ }^{15}$, spermicidal and anti-HIV ${ }^{16}, \beta$-adrenergic receptor antagonist properties ${ }^{17}$. Similarly the third pharmacophore methylsulfanyl oxadiazole unit is also one of important biologically active building block. The substituted methylsulfanyloxadiazoles have been found to exhibit diverse biological activities such as inhibitors of glycogen synthase kinase- $3 \beta^{18}$, antifungal ${ }^{19}$, antibacterial and anti-HIV ${ }^{20}$, insecticidal ${ }^{21}$, antiinflammatory and analgesic ${ }^{22}$, anticonvulsants and muscle relaxants ${ }^{23}$.

The diverse biological activities of these pharmacophores; phthalazin-1(2H)-one, methylsulfanyl oxadiazole and isoxazoline pharmacophores, encouraged us to discover a new lead compounds, which contains all these pharmocophores in a single molecules, that may exhibit higher pharmacological activities. By combining these pharmacophore components in a single molecule, to give a compact system, we designed and synthesized a series of phthalazin-1(2H)-one derivatives containing methylsulfanyl oxadiazole and isoxazoline moieties. The synthesized new phthalazin-1(2H)-one derivatives were characterized by mass, IR and NMR spectral data's.

\section{Experimental}

NMR spectra were recorded on $400 \mathrm{MHz}$ Varian-AS NMR spectrometer using TMS as an internal standard. IR spectra were recorded by using Perkin Elmer Spectrum 100 Series FT-IR spectrometer. Mass spectra were recorded on Agilent 1200 Series LC/MSD VL system. Melting points were determined by using Buchi melting point B-545 instrument and are uncorrected. All reactions were monitored by thin layer chromatography (TLC) using Merck pre-coated TLC silica gel plates. The crude compounds were purified by using CombiFlash ${ }^{\circledR}$ Companion ${ }^{\circledR}$ flash chromatography system, Teledyne Isco, Inc USA. BPL Sanyo domestic microwave oven was used for reactions.

Preparation of methyl 2-(3-allyl-4-oxo-3,4-dihydrophthalazin-1-yl)acetate (2)

Allyl bromide $(60.5 \mathrm{~g}, 0.5 \mathrm{~mol})$ was added to a stirred solution of methyl 2-(4-oxo-3,4dihydrophthalazin-1-yl)acetate $(\mathbf{1})^{24}(21.8 \mathrm{~g}, 0.10 \mathrm{~mol})$ in dimethylformamide $(250 \mathrm{~mL})$ and 
potassium carbonate $(69.0 \mathrm{~g}, 0.5 \mathrm{~mol})$ at room temperature and heated to $60-65{ }^{\circ} \mathrm{C}$ for $6 \mathrm{~h}$. After completion of reaction, filtered the inorganics, the filtrate obtained was distilled completely under reduced pressure at $60-65{ }^{\circ} \mathrm{C}$. The residue obtained was diluted with ice water $(500 \mathrm{~mL})$ and stirred for 30 minutes. The precipitated product was filtered, dried and recrystallized using isopropyl alcohol to yield compound $\mathbf{2}$ as white solid. M.p.: $90.2-95.8{ }^{\circ} \mathrm{C}$; MS: $m / z=259.1\left(\mathrm{M}^{+}+1\right)$; IR $(\mathrm{KBr}) \vee \mathrm{cm}^{-1}: 3012,1708,1675,1599 ;{ }^{1} \mathrm{H}$ NMR $(400 \mathrm{MHz}$, DMSO-d $\left.\mathrm{d}_{6}\right) \delta: 3.64\left(\mathrm{~s}, 3 \mathrm{H}, \mathrm{CH}_{3}\right), 4.11\left(\mathrm{~s}, 2 \mathrm{H}, \mathrm{CH}_{2}\right), 4.73-4.74\left(\mathrm{~d}, \mathrm{~J}=5.6 \mathrm{~Hz}, \mathrm{CH}_{2}\right), 5.08-5.19$ (m, $\left.2 \mathrm{H}, \mathrm{CH}_{2}\right)$, 5.94-6.04 (m, 1H, $\left.\mathrm{CH}_{2}\right), 7.87-7.92(\mathrm{~m}, 3 \mathrm{H}, \mathrm{Ar}-\mathrm{H}), 8.30-8.32(\mathrm{~m}, 1 \mathrm{H}, \mathrm{Ar}-\mathrm{H})$.

\section{Preparation of 2-(3-allyl-4-oxo-3,4-dihydrophthalazin-1-yl)acetohydrazide (3)}

To a solution of phthalazin-1(2H)-one acetic acid methyl ester $(2),(20.0 \mathrm{~g}, 0.077 \mathrm{~mol})$ in ethanol $(300 \mathrm{~mL})$ was added hydrazine hydrate $(11.5 \mathrm{~g}, 0.22 \mathrm{~mol})$. The reaction mixture was heated under reflux for $10 \mathrm{~h}$. Cooled the reaction mixture to room temperature, the precipitate obtained was filtered, dried to yield compound $3(11.5 \mathrm{~g})$ as white solid. Yield: $74.6 \%$; M.p.: 201.4-207.9 ${ }^{\circ} \mathrm{C}$; MS: $m / z=259.1(\mathrm{M}+1)$; IR $(\mathrm{KBr}) \vee \mathrm{cm}^{-1}: 3202,1758,1673 ;{ }^{1} \mathrm{H}$ NMR $(400$ MHz, DMSO-d $\left.{ }_{6}\right): \delta{ }^{1} \mathrm{H}$ NMR (DMSO-d $\left.{ }_{6}, 400 \mathrm{MHz}\right) \delta: 3.75\left(\mathrm{~s}, 2 \mathrm{H}, \mathrm{CH}_{2}\right), 4.26-4.27(\mathrm{~d}, J=4.0$ $\left.\mathrm{Hz}, 2 \mathrm{H}, \mathrm{CH}_{2}\right), 4.72-4.73\left(\mathrm{~d}, J=5.6 \mathrm{~Hz}, 2 \mathrm{H}, \mathrm{NH}_{2}\right), 5.11-5.19\left(\mathrm{~m}, 2 \mathrm{H}, \mathrm{CH}_{2}\right), 5.94-6.04(\mathrm{~m}, 1 \mathrm{H}$, $\mathrm{CH}), 7.85-7.94(\mathrm{~m}, 3 \mathrm{H}, \mathrm{Ar}-\mathrm{H}), 8.28-8.30(\mathrm{~d}, J=8.0,1 \mathrm{H}, \mathrm{Ar}-\mathrm{H}), 9.32(\mathrm{~s}, 1 \mathrm{H}, \mathrm{NH})$.

\section{Preparation of 2-allyl-4-((5-mercapto-1,3,4-oxadiazol-2-yl)methyl)phthalazin-1 (2H)-one (4)}

Compound 3 (11.0 g, $0.042 \mathrm{~mol})$ was added to a solution of $\mathrm{KOH}(3.8 \mathrm{~g}, 0.07 \mathrm{~mol})$ in anhydrous ethanol $(160 \mathrm{~mL})$. A solution of $\mathrm{CS}_{2}(8.2 \mathrm{~g}, 0.113 \mathrm{~mol})$ in anhydrous ethanol $(40 \mathrm{~mL})$ was added drop wise to the vigorously stirred mixture, which was refluxed for $10 \mathrm{~h}$. After completion of reaction, cooled and evaporated the solvent under reduced pressure. The residue obtained was dissolved in water $(200 \mathrm{~mL})$ and acidified to $\mathrm{pH} 5 \sim 6$ with hydrochloric acid. The precipitated product was isolated by filtration and drying to yield compound 4 $(8.5 \mathrm{~g})$ as white solid. Yield: $66.6 \%$; M.p.: $195.2-199.3{ }^{\circ} \mathrm{C}$; $\mathrm{MS}: m / z=301.1\left(\mathrm{M}^{+}+1\right)$; IR $(\mathrm{KBr}) \mathrm{vcm}^{-1}: 2540,1754,1672 ;{ }^{1} \mathrm{H}$ NMR $\left(400 \mathrm{MHz}, \mathrm{DMSO}-\mathrm{d}_{6}\right) \delta: 4.59\left(\mathrm{~s}, 2 \mathrm{H}, \mathrm{CH}_{2}\right), 4.70-$ 4.72(d, J=5.6 Hz, 2H, CH$\left.)_{2}\right), 5.09-5.19\left(\mathrm{~m}, 2 \mathrm{H}, \mathrm{CH}_{2}\right), 5.92-6.02(\mathrm{~m}, 1 \mathrm{H}, \mathrm{CH}), 7.88-8.27$ (m, 3H, Ar-H), 8.27-8.33 (m, 1H, Ar-H), 14.37 (s, 1H, SH).

Preparation of 2-allyl-4-((5-(methylthio)-1,3,4-oxadiazol-2-yl)methyl) phthalazin$1(2 \mathrm{H})$ - one $(5)$

The compound $4(8.0 \mathrm{~g}, 0.026 \mathrm{~mol})$ was dissolved in $10 \%$ potassium hydroxide in water $(100 \mathrm{~mL})$. To the clear solution, methyl iodide $(4.4 \mathrm{~g}, 0.03 \mathrm{~mol})$ was added and stirred for $4 \mathrm{~h}$ at room temperature. After completion of reaction (checked by TLC), precipitated product was filtered, washed with water, dried to get compound $5(7.5 \mathrm{~g})$ as white solid. Yield: 88.3\%; M.p.: $119.9-125.5^{\circ} \mathrm{C}$; MS: $m / z=315.1\left(\mathrm{M}^{+}+1\right)$; IR $(\mathrm{KBr}) \mathrm{cm}^{-1}: 2935,1711,1652 ;{ }^{1} \mathrm{H}$ NMR (400 MHz, DMSO-d $\left.{ }_{6}\right) \delta: 2.60\left(\mathrm{~s}, 3 \mathrm{H}, \mathrm{S}_{-} \mathrm{CH}_{3}\right), 4.69\left(\mathrm{~s}, 2 \mathrm{H}, \mathrm{CH}_{2}\right), 4.71-4.72$ (d, J=5.6 $\left.\mathrm{Hz}, 2 \mathrm{H}, \mathrm{CH}_{2}\right)$ 5.07-5.18 (m, 2H, $\left.\mathrm{CH}_{2}\right), 5.91-6.00(\mathrm{~m}, 1 \mathrm{H}, \mathrm{CH}), 7.89-8.03(\mathrm{~m}, 3 \mathrm{H}, \mathrm{Ar}-\mathrm{H})$, 8.31-8.33 (d, J=7.6 Hz, 1H, Ar-H).

General method for the preparation of 3-substituted phthalazin-1(2H)-one derivatives $\mathbf{6}(\boldsymbol{a}-\boldsymbol{i})$

An equimolar mixture of aldoxime $(10.0 \mathrm{mmol})$ and $N$-chlorosuccinimide $(10.0 \mathrm{mmol})$ were made paste with dimethylformamide $(2 \mathrm{~mL})$ and alumina $(2 \mathrm{~g})$ in a $10 \mathrm{~mL}$ beaker. The mixture was irradiated at $600 \mathrm{~W}$ for $2 \mathrm{~min}$ in domestic microwave oven. After adding 
compound (5) $(9 \mathrm{mmol})$, the reaction mixture was irradiated further for $6 \mathrm{~min}$. After completion of reaction, diluted with water $(25 \mathrm{~mL})$ and extracted with ethyl acetate $(3 \times 50 \mathrm{~mL})$. The organic layer was washed with water $(10 \mathrm{~mL})$ and then with saturated sodium chloride solution. After drying with anhydrous sodium sulphate and filtration, the solvent was evaporated to get crude product, which was purified by using flash chromatography eluting with ethyl acetate / hexane (1:5) to get the corresponding phthalazin-1(2H)-ones 6(a-i).

2-((3-Methyl-4,5-dihydroisoxazol-5-yl)methyl)-4-((5-(methylthio)-1,3,4-oxadiazol2-yl)methyl)phthalazin-1(2H)-one (6a)

White solid; Yield: 52.1\%; M.p.: $119.3-121.9{ }^{\circ} \mathrm{C}$; MS: $\mathrm{m} / \mathrm{z}=372.1\left(\mathrm{M}^{+}+1\right)$; ${ }^{1} \mathrm{H}$ NMR $\left(400 \mathrm{MHz}, \mathrm{DMSO}-\mathrm{d}_{6}\right) \delta: 1.92\left(\mathrm{~s}, 3 \mathrm{H}, \mathrm{C}-\mathrm{CH}_{3}\right), 2.65\left(\mathrm{~s}, 3 \mathrm{H}, \mathrm{S}-\mathrm{CH}_{3}\right), 3.29-3.45\left(\mathrm{~m}, 2 \mathrm{H}, \mathrm{CH}_{2}\right)$, 4.34-4.39 (dd, $\left.J=6.8 \mathrm{~Hz}, 13.4 \mathrm{~Hz}, 1 \mathrm{H}, \mathrm{CH}_{2}\right), 4.45-4.55\left(\mathrm{~m}, 3 \mathrm{H}\right.$, two $\left.\mathrm{CH}_{2}\right), 5.22-5.26(\mathrm{~m}, 1 \mathrm{H}$, oxazole-CH), 7.61-7.91 (m, 3H, Ar-H), 8.29-8.31 (m, 1H, Ar-H); ${ }^{13} \mathrm{C}$ NMR (400 MHz, DMSO-d $\left.{ }_{6}\right) \delta: 163.7,159.7,156.1,139.9,141.9,140.0,134.35,132.9,132.7,128.9,127.6$, 127.0, 126.0, 124.7, 50.9, 29.1, 10.68; IR (KBr) cm $\mathrm{cm}^{-1}: 2937,1712,1658,1514$.

4-((5-(Methylthio)-1,3,4-oxadiazol-2-yl)methyl)-2-((3-phenyl-4,5-dihydroisoxazol5-yl)methyl)phthalazin-1(2H)-one (6b)

Yield: 65.6\%; M.p.: $144.6-148.3{ }^{\circ} \mathrm{C}$; MS: $\mathrm{m} / z=434.1\left(\mathrm{M}^{+}+1\right) ;{ }^{1} \mathrm{H}$ NMR $\left(400 \mathrm{MHz}\right.$, DMSO-d $\left.{ }_{6}\right)$ $\delta: 2.65\left(\mathrm{~s}, 3 \mathrm{H}, \mathrm{S}-\mathrm{CH}_{3}\right), 3.29-3.45\left(\mathrm{~m}, 2 \mathrm{H}, \mathrm{CH}_{2}\right), 4.34-4.39$ (dd, $\left.J=6.8,13.4 \mathrm{~Hz}, 1 \mathrm{H}, \mathrm{CH}_{2}\right)$, 4.45-4.55 (m, 3H, two $\left.\mathrm{CH}_{2}\right), 5.22-5.26(\mathrm{~m}, 1 \mathrm{H}$, oxazole- $\mathrm{CH}), 7.38-7.39(\mathrm{~m}, 3 \mathrm{H}, \mathrm{Ar}-\mathrm{H}), 7.67-$ $7.87(\mathrm{~m}, 5 \mathrm{H}, \mathrm{Ar}-\mathrm{H}), \quad 8.45-8.47(\mathrm{~d}, J=7.2 \mathrm{~Hz}, 1 \mathrm{H}, \mathrm{Ar}-\mathrm{H}) ;{ }^{13} \mathrm{C}$ NMR $(400 \mathrm{MHz}$, DMSO-d 6 ) $\delta$ : $166.1,163.9,159.5,156.5,139.9,133.6,132.0,130.1,129.2,128.6,128.5,127.5,126.8$, 124.3, 78.1, 77.4, 77.0, 76.7, 53.5, 38.4, 29.7, 14.4; IR (KBr) cm${ }^{-1}: 2998,1673,1532$.

2-((3-(4-Chlorophenyl)-4,5-dihydroisoxazol-5-yl)methyl)-4-((5-(methylthio)1,3,4-oxadiazol-2-yl)methyl)phthalazin-1(2H)-one (6c)

White solid; Yield: 53.2\%; M.p.: 122.3-127.9 ${ }^{\circ} \mathrm{C}$; MS: $m / z=468.1\left(\mathrm{M}^{+}+1\right) ;{ }^{1} \mathrm{H}$ NMR $(400 \mathrm{MHz}$, DMSO-d $_{6}$ ): $\delta 2.67\left(\mathrm{~s}, 3 \mathrm{H}, \mathrm{S}-\mathrm{CH}_{3}\right), 3.28-3.43\left(\mathrm{~m}, 2 \mathrm{H}, \mathrm{CH}_{2}\right), 4.32-4.37$ (dd, J=6.8, $13.4 \mathrm{~Hz}$, $\left.1 \mathrm{H}, \mathrm{CH}_{2}\right), 4.48-4.58\left(\mathrm{~m}, 3 \mathrm{H}\right.$, two $\left.\mathrm{CH}_{2}\right), 5.23-5.27(\mathrm{~m}, 1 \mathrm{H}$, oxazole- $\mathrm{CH}), 7.34-7.36(\mathrm{~d}, J=8.0 \mathrm{~Hz}$, 2H, Ar-H), 7.76-7.88 (m, 3H, Ar-H), 8.43-8.45 (d, J=7.6 Hz, 1H, Ar-H); ${ }^{13} \mathrm{C}$ NMR (400 MHz, DMSO-d $\left._{6}\right) \delta: 166.0,163.9,159.4,155.6,140.0,136.0,133.7,132.0,129.1,128.6,128.0,127.7$, 127.4, 124.3, 78.4, 77.4, 77.1, 76.8, 53.3, 38.2, 29.6, 14.4; IR (KBr) cm ${ }^{-1}: 2989,1681,1542$.

2-((3-(4-Fluorophenyl)-4,5-dihydroisoxazol-5-yl)methyl)-4-((5-(methylthio)1,3,4-oxadiazol-2-yl)methyl)phthalazin-1(2H)-one (6d)

Yield: 53.8\%; White solid; M.p.: $130.2-133-8{ }^{\circ} \mathrm{C} ; \quad \mathrm{MS}: \mathrm{m} / z=452.1\left(\mathrm{M}^{+}+1\right) ;{ }^{1} \mathrm{H}$ NMR (400 MHz, DMSO-d $\left.\mathrm{d}_{6}\right) \delta: 2.67$ (s, 3H, S-CH $\left.\mathrm{CH}_{3}\right), 3.28-3.43\left(\mathrm{~m}, 2 \mathrm{H}, \mathrm{CH}_{2}\right), 4.32-4.37$ (dd, J=6.8, $\left.13.4 \mathrm{~Hz}, 1 \mathrm{H}, \mathrm{CH}_{2}\right), 4.48-4.58\left(\mathrm{~m}, 3 \mathrm{H}\right.$, two $\left.\mathrm{CH}_{2}\right), 5.23-5.27$ (m, 1H, oxazole- $\left.\mathrm{CH}\right), 7.34-7.36$ $(\mathrm{d}, J=8.0 \mathrm{~Hz}, 2 \mathrm{H}, \mathrm{Ar}-\mathrm{H}), 7.56-7.62(\mathrm{~d}, J=8.0 \mathrm{~Hz}, 2 \mathrm{H}, \mathrm{Ar}-\mathrm{H}), 7.76-7.88$ (m, 3H, Ar-H), 8.43$8.45(\mathrm{~d}, J=7.6 \mathrm{~Hz}, 1 \mathrm{H}, \mathrm{Ar}-\mathrm{H}) ;{ }^{13} \mathrm{C}$ NMR $\left(400 \mathrm{MHz}, \mathrm{DMSO}-\mathrm{d}_{6}\right) \delta: 166.0,163.9,159.4$, 155.6, 140.0, 136.0, 1133.7, 132.0, 129.1, 128.5, 128.0, 127.7, 127.4, 124.3, 78.4, 77.4, 77.1, 76.8, 53.3, 38.2, 29.6, 14.4; IR (KBr) cm $\mathrm{cm}^{-1}$ : 2989, 1752, 1681, 1542.

2-((3-(4-Methoxyphenyl)-4,5-dihydroisoxazol-5-yl)methyl)-4-((5-(methylthio)1,3,4-oxadiazol-2-yl)methyl)phthalazin-1(2H)-one (6e)

Yield: 59.3\%; White solid; M.p.: 151.6-155.8 ${ }^{\circ} \mathrm{C}$; MS: $m / z=464.1\left(\mathrm{M}^{+}+1\right)$; ${ }^{1} \mathrm{H}$ NMR (400 MHz, DMSO-d $\left.)_{6}\right) \delta: 2.65\left(\mathrm{~s}, 3 \mathrm{H}, \mathrm{S}-\mathrm{CH}_{3}\right), 3.27-3.42\left(\mathrm{~m}, 2 \mathrm{H}, \mathrm{CH}_{2}\right), 3.82\left(\mathrm{~s}, 3 \mathrm{H},-\mathrm{OCH}_{3}\right)$, 
4.30-4.35 (dd, $\left.J=6.4 \mathrm{~Hz}, 1 \mathrm{H}, \mathrm{CH}_{2}\right), 4.46-4.52\left(\mathrm{~m}, 3 \mathrm{H}\right.$, two $\left.\mathrm{CH}_{2}\right), 5.18-5.21$ (m, 1H, oxazole- $\left.\mathrm{CH}\right)$, $6.88(\mathrm{dd}, J=8.8 \mathrm{~Hz}, 2 \mathrm{H}, \mathrm{Ar}-\mathrm{H}), 7.58-7.60(\mathrm{~d}, J=8.8 \mathrm{~Hz}, 2 \mathrm{H}, \mathrm{Ar}-\mathrm{H}), 7.75-7.87(\mathrm{~m}, 3 \mathrm{H}$, Ar-H), 8.42 (d, J=7.6 Hz, 1H, Ar-H); ${ }^{13} \mathrm{C}$ NMR (400 MHz, DMSO-d $\left.{ }_{6}\right) \delta: 166.0,163.9$, $161.0,159.4,156.1,139.9,133.6,131.9,129.3,128.2,127.3,124.3,122.3,114.0,77.5$, 76.9, 55.3, 53.4, 38.6, 36.3, 29.6, 14.4; IR (KBr) cm $\mathrm{cm}^{-1}$ : 2988, 1753, 1657, 1545.

2-((3-(3,4-Dimethoxyphenyl)-4,5-dihydroisoxazol-5-yl)methyl)-4-((5-(methylthio)1,3,4-oxadiazol-2-yl)methyl)phthalazin-1(2H)-one (6f)

Yield: 45.0\%; White solid; M.p.: 146.0-149.8 ${ }^{\circ} \mathrm{C}$; MS $m / z=494.1\left(\mathrm{M}^{+}+1\right)$; ${ }^{1} \mathrm{H}$ NMR $\left(400 \mathrm{MHz}, \mathrm{DMSO}-\mathrm{d}_{6}\right) \delta$ : $2.68\left(\mathrm{~s}, 3 \mathrm{H}, \mathrm{S}-\mathrm{CH}_{3}\right), 3.25-3.45\left(\mathrm{~m}, 1 \mathrm{H}, \mathrm{CH}_{2}\right), 3.74\left(\mathrm{~m}, 1 \mathrm{H}, \mathrm{CH}_{2}\right)$, $3.91\left(\mathrm{~s}, 6 \mathrm{H}\right.$, two $\left.-\mathrm{OCH}_{3}\right), 4.37-4.48\left(\mathrm{dd}, J=6.4 \mathrm{~Hz}, 1 \mathrm{H}, \mathrm{CH}_{2}\right), 4.52-4.57\left(\mathrm{~m}, 3 \mathrm{H}\right.$, two $\left.\mathrm{CH}_{2}\right)$, 5.18-5.24 (m, 1H, oxazole-CH), 6.93-6.95 (dd, $J=8.0 \mathrm{~Hz}, 2 \mathrm{H}, \mathrm{Ar}-\mathrm{H}), 7.05-7.14$ (d, $J=8.8 \mathrm{~Hz}$, 2H, Ar-H), 7.38-7.40 (m, 1H, Ar-H), 7.57-7.7.99 (m, 2H, Ar-H), 8.47-8.49 (d, J=7.2 Hz, $1 \mathrm{H}, \mathrm{Ar}-\mathrm{H}) ;{ }^{13} \mathrm{C}$ NMR (400 MHz, DMSO-d 6 ) $\delta: 166.0,163.9,161.0,159.4,156.1,139.9$, 133.6, 131.9, 129.3, 128.4, 128.2, 127.3, 124.3, 122.3, 121.6, 114.0, 77.7, 77.5, 77.2, 76.9, 55.3, 53.4, 38.6, 36.3, 29.6, 14.4; IR (KBr) cm ${ }^{-1}: 2979,17650,1673,1545$.

2-((3-(3-(4-Fluorophenoxy)phenyl)-4,5-dihydroisoxazol-5-yl)methyl)-4-(5-methylthio)1,3,4-oxadiazol-2-yl)methyl)phthalazin-1(2H)-one (6g)

Yield: 52.8\%; White solid; M.p.: 171.1-174.9 ${ }^{\circ} \mathrm{C}$; MS: $\mathrm{m} / \mathrm{z}=544.1\left(\mathrm{M}^{+}+1\right) ;{ }^{1} \mathrm{H}$ NMR (400 MHz, DMSO-d $\left.{ }_{6}\right) \delta: 2.66\left(\mathrm{~s}, 3 \mathrm{H}, \mathrm{S}-\mathrm{CH}_{3}\right), 3.24-3.40\left(\mathrm{~m}, 2 \mathrm{H}, \mathrm{CH}_{2}\right), 4.34-4.39$ (dd, J=6.8, $\left.13.4 \mathrm{~Hz}, 1 \mathrm{H}, \mathrm{CH}_{2}\right)$, 4.45-4.56 (m, $\left.3 \mathrm{H}, \mathrm{CH}_{2}\right), 5.20-5.34(\mathrm{~m}, 1 \mathrm{H}$, oxazole- $\mathrm{CH}), 6.98-7.00$ $(\mathrm{d}, J=7.6 \mathrm{~Hz}, 2 \mathrm{H}, \mathrm{Ar}-\mathrm{H}), 7.09-7.11(\mathrm{t}, J=7.6,7.2 \mathrm{~Hz}, 1 \mathrm{H}, \mathrm{Ar}-\mathrm{H}), 7.21-7.23(\mathrm{~m}, 1 \mathrm{H}, \mathrm{Ar}-\mathrm{H})$, 7.33-7.45 (m, 4H, Ar-H), 7.78-7.88 (m, 3H, Ar-H), 8.45-8.47 (m, 1H, Ar-H); ${ }^{13} \mathrm{C} \mathrm{NMR}$ (400 MHz, DMSO-d ${ }_{6}$ ) $\delta: 166.1,163.9,159.5,156.7,155.2,153.9,144.2,139.9,133.7$, 132.0, 129.8, 128.5, 127.8, 127.5, 126.4, 123.6, 123.3, 119.7, 117.6, 117.2, 78.4, 77.4, 77.1, 76.8, 53.4, 38.3, 29.6, 14.1; IR (KBr) cm $\mathrm{cm}^{-1}$ :2979, 1775, 1673, 1544.

4-((5-(Methylthio)-1,3,4-oxadiazol-2-yl)methyl)-2-((3-(thiophen-2-yl)-4,5-dihydro isoxazol-5-yl)methyl)phthalazin-1(2H)-one (6h)

Yield: 70.2\%; Off-white solid; M.p.: $139.5-143.1{ }^{\circ} \mathrm{C}$; MS: $\mathrm{m} / \mathrm{z}=440.1\left(\mathrm{M}^{+}+1\right) ;{ }^{1} \mathrm{H}$ NMR $\left(400 \mathrm{MHz}, \mathrm{DMSO}-\mathrm{d}_{6}\right) \delta: 1.091 .23\left(\mathrm{~m}, 1 \mathrm{H}, \mathrm{CH}_{2}\right), 2.66\left(\mathrm{~s}, 3 \mathrm{H}, \mathrm{S}-\mathrm{CH}_{3}\right), 3.51-3.58(\mathrm{dd}, J=10.4$, $\left.16.8 \mathrm{~Hz}, 1 \mathrm{H}, \mathrm{CH}_{2}\right), 4.05-4.13\left(\mathrm{~m}, 1 \mathrm{H}, \mathrm{CH}_{2}\right), 4.41-4.54\left(\mathrm{~m}, 1 \mathrm{H}, \mathrm{CH}_{2}\right), 4.71-4.87(\mathrm{~m}, 2 \mathrm{H}$, $\left.\mathrm{CH}_{2}\right), 5.08-5.12(\mathrm{~m}, 1 \mathrm{H}$, Oxazole $\mathrm{CH}), 7.17-7.22(\mathrm{~m}, 1 \mathrm{H}, \mathrm{Ar}-\mathrm{H}), 7.38-7.39(\mathrm{~d}, J=3.6 \mathrm{~Hz}, 1 \mathrm{H}$, Ar-H), 7.70-7.71 (d, J=4.8 Hz, 1H, Ar-H), 7.85-8.15 (m, 3H, Ar-H), 8.32-8.34 (d, J=7.6 Hz, $1 \mathrm{H}, \mathrm{Ar}-\mathrm{H}) .{ }^{13} \mathrm{C}$ NMR $\left(400 \mathrm{MHz}, \mathrm{DMSO}-\mathrm{d}_{6}\right) \delta: 159.4,155.6,140.0,136.0,133.7,132.0$, 129.1, 128.9, 128.6, 128.4, 127.7, 127.7, 124.3, 77.4, 77.1, 76.8, 53.4, 38.3, 29.6, 14.1; IR $(\mathrm{KBr}) \mathrm{cm}^{-1}: 2989,1760,1681,1542$.

2-((3-(2-Hydroxyphenyl)-4,5-dihydroisoxazol-5-yl)methyl)-4-((5-(methylthio)1,3,4-oxadiazol-2-yl)methyl)phthalazin-1(2H)-one (6i)

Yield: 45.6\%; White solid; M.p.: 169.2-172.9 ${ }^{\circ} \mathrm{C}$; MS: $\mathrm{m} / \mathrm{z}=450.1\left(\mathrm{M}^{+}+1\right) ;{ }^{1} \mathrm{H}$ NMR (400 MHz, DMSO-d 6 ) $\delta: 2.66\left(\mathrm{~s}, 3 \mathrm{H}, \mathrm{S}_{-} \mathrm{CH}_{3}\right), 3.41-3.48\left(\mathrm{~m}, 1 \mathrm{H}, \mathrm{CH}_{2}\right), 3.60-3.67$ (dd, $\left.J=10.4,17.2 \mathrm{~Hz}, 1 \mathrm{H}, \mathrm{CH}_{2}\right), 4.16-4.20\left(\mathrm{dd}, J=5.6,13.6 \mathrm{~Hz}, 1 \mathrm{H}, \mathrm{CH}_{2}\right), 4.39-4.44$ (q, J=7.6, $\left.13.4 \mathrm{~Hz}, 1 \mathrm{H}, \mathrm{CH}_{2}\right), 4.66-4.75\left(\mathrm{~m}, 2 \mathrm{H}, \mathrm{CH}_{2}\right), 5.07-5.11(\mathrm{~m}, 1 \mathrm{H}$, oxazole- $\mathrm{CH}), 6.90-6.97$ (q, $J=7.2,8.4,17.8 \mathrm{~Hz}, 2 \mathrm{H}, \mathrm{Ar}-\mathrm{H}) .7 .31-7.35(\mathrm{t}, J=7.2,7.6 \mathrm{~Hz}, 1 \mathrm{H}, \mathrm{Ar}-\mathrm{H}), 7.42-7.44$ (d, J=7.6 Hz, 1H, Ar-H), 7.90-8.05 (m, 3H, Ar-H), 8.32-8.34 (d, J=8.0 Hz, 1H, Ar-H), 9.84 $(\mathrm{s}, 1 \mathrm{H}, \mathrm{OH}) ;{ }^{13} \mathrm{C}$ NMR $\left(400 \mathrm{MHz}, \mathrm{DMSO}-\mathrm{d}_{6}\right) \delta: 166.1,163.9,159.5,156.5,139.9,133.6$, 132.0, 130.1, 129.2, 128.6, 128.5, 127.5, 126.8, 124.3, 78.1, 77.4, 77.0, 76.7, 53.5, 38.4, 29.7, 14.4; IR (KBr) cm $\mathrm{cm}^{-1}: 3326,2975,1675$. 


\section{Results and Discussion}

The key intermediate in the synthesis of new phthalazine- $1(2 H)$-one isoxazolines $\mathbf{6}(\mathbf{a}-\mathbf{i})$ is methyl 2-(4-oxo-3,4-dihydrophthalazin-1-yl)acetate (1), which was prepared from phthalic anhydride in good yield ${ }^{24}$.<smiles>COC(=O)Cc1n[nH]c(=O)c2ccccc12</smiles><smiles>C=CCn1nc(CC(=O)OC)c2ccccc2c1=O</smiles><smiles>C=CCn1nc(CC(=O)NN)c2ccccc2c1=O</smiles><smiles>[R]C1=NOC(Cn2nc(Cc3nnc(S)o3)c3ccccc3c2=O)C1</smiles>

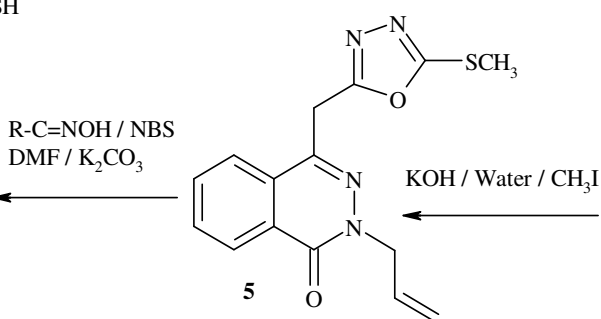<smiles>C=CCn1nc(Cc2nnc(S)o2)c2ccccc2c1=O</smiles>

$R=a)$ Methyl, b) Phenyl, C) 4-Chlorophenyl, d) 4-Fluorophenyl, e) 4-methoxyphenyl, f) 3-4Dimethoxyphenyl, g) 4-Fluoro(3-phenoxy)phenyl, h) Thiophene-2yl, i) 2-hydroxyphenyl

Scheme 1. Synthesis of phthalazine isoxazoline derivatives 6(a-i)

$\mathrm{N}$-allylation of compound (1) with allyl bromide in presence of potassium carbonate gave methyl 2-(3-allyl-4-oxo-3,4-dihydrophthalazin-1-yl)acetate (2). The compound 2 on reaction with hydrazine hydrate afford hydrazide 3. Oxadiazolinethione (4) was prepared from reaction of the hydrazide $\mathbf{3}$ with carbon disulphide and potassium hydroxide, in ethanol, followed by acidification. On treatment with methyl iodide, the compound (4) gave 2-allyl-4-(5-mercapto-1,3,4-oxadiazol-2-yl)methyl)phthalazin-1(2H)-one (5). Allyl function of compound (5) undergoes 1,3 dipolar cyclization ${ }^{25}$ with aldoximes ${ }^{26}$ via nitrile oxide in presence of $\mathrm{N}$-chlorosuccinimide, potassium carbonate and dimethylformamide gave desired compounds 6(a-i). The synthesised new compounds were characterised by mass, IR, NMR and elemental analysis studies.

\section{Biological screening}

The in vitro antimicrobial activity was carried out against $24 \mathrm{~h}$ old cultures of two bacteria and two fungi by cup-plate method in duplicate. Compounds (6a-i) has been tested for their antimicrobial activity against $E$. coli and $S$. aureus and antifungal activity against $M$. gypsum and A. flavus at a concentration of $100 \mu \mathrm{g} / \mathrm{mL}$ in DMSO using cup plate diffusion method. Nutrient agar and potato dextrose agars were used to culture the bacteria and fungus respectively. The solution of Amoxicillin $100 \mu \mathrm{g} / \mathrm{mL}$ and metronidazole at $100 \mu \mathrm{g} / \mathrm{mL}$ were prepared in DMSO and used as standard for comparison of antibacterial and antifungal activities respectively the results were discussed in Table 1. 
Table 1. Antimicrobial activity of compounds 6(a-i)

\begin{tabular}{|c|c|c|c|c|}
\hline \multirow{4}{*}{$\begin{array}{l}\text { Synthesized } \\
\text { compounds }\end{array}$} & \multicolumn{4}{|c|}{ Zone of Inhibition measured in $\mathrm{mm}$} \\
\hline & \multicolumn{2}{|c|}{ Antibacterial activity } & \multicolumn{2}{|c|}{ Antifungal activity } \\
\hline & $\begin{array}{c}\text { Escherichia } \\
\text { Coli } \pm \text { S.D }\end{array}$ & $\begin{array}{c}\text { Staphylococcus } \\
\text { Aureus } \pm \text { S.D }\end{array}$ & $\begin{array}{l}\text { Microsporum } \\
\text { Gypsum } \pm \text { S.D }\end{array}$ & $\begin{array}{l}\text { Aspergillus } \\
\text { flavus }^{\mathrm{d}} \pm \mathrm{S} . \mathrm{D}\end{array}$ \\
\hline & $100 \mu \mathrm{g} / \mathrm{mL}$ & $100 \mu \mathrm{g} / \mathrm{mL}$ & $100 \mu \mathrm{g} / \mathrm{mL}$ & $100 \mu \mathrm{g} / \mathrm{mL}$ \\
\hline $6 \mathbf{6 a}$ & $2.50 \pm 0.70$ & $4.50 \pm 0.70$ & $4.50 \pm 0.70$ & $4.50 \pm 0.70$ \\
\hline 6b & $6.50 \pm 0.70$ & $8.5 \pm 0.70$ & $3.50 \pm 0.70$ & $11.50 \pm 0.70$ \\
\hline $6 c$ & $5.00 \pm 0.00$ & $7.5 \pm 0.70$ & $4.50 \pm 0.70$ & $7.50 \pm 0.70$ \\
\hline 6d & $3.00 \pm 0.00$ & $4.50 \pm 0.70$ & $5.50 \pm 0.70$ & $5.50 \pm 0.70$ \\
\hline $6 e$ & $3.50 \pm 0.70$ & $3.00 \pm 0.00$ & $3.00 \pm 0.00$ & $5.00 \pm 0.00$ \\
\hline 6f & $7.50 \pm 0.70$ & $8.00 \pm 0.00$ & $2.50 \pm 0.70$ & $7.50 \pm 0.70$ \\
\hline $6 g$ & $8.50 \pm 0.70$ & $11.50 \pm 0.70$ & $7.00 \pm 0.00$ & $11.00 \pm 0.00$ \\
\hline $6 h$ & $6.00 \pm 0.70$ & $7.50 \pm 0.70$ & $4.50 \pm 0.70$ & $4.50 \pm 0.70$ \\
\hline 6i & $3.00 \pm 0.00$ & $3.00 \pm 0.00$ & $3.00 \pm 0.00$ & $5.00 \pm 0.00$ \\
\hline Metronidazole & - & - & $17.50 \pm 0.70$ & $17.50 \pm 0.70$ \\
\hline Amoxicillin & $9.00 \pm 0.00$ & $15.50 \pm 0.70$ & - & - \\
\hline
\end{tabular}

The compounds $\boldsymbol{\sigma} \boldsymbol{b}$ and $\boldsymbol{\sigma} \boldsymbol{f}-\boldsymbol{g}$ exhibiting good activity against E. coli and compounds $\boldsymbol{\sigma} \boldsymbol{b}, \boldsymbol{\sigma} \boldsymbol{c}, \boldsymbol{\sigma} \boldsymbol{f}$ and $\boldsymbol{\sigma} \boldsymbol{g}$ show good activity of against $S$. Aureus. Similarly the compounds $7 \mathrm{~g}$ showed good activity against M.gypsum and the compounds $\boldsymbol{\sigma} \boldsymbol{b}$ and $\boldsymbol{6} \boldsymbol{g}$ showed activity against A. and all remaining compounds exhibiting moderate activity against all the four organisms used for screening

\section{Conclusion}

In this article we report the synthesis of (6a-i), new phthalazine isoxazolines substituted at $\mathrm{C}_{4}$ position with methylsulfonyl oxadiazole starting from commercially available phthalic anhydride. Investigation of their antimicrobial activity revealed that isoxazolines with substitution with thiophene (6h) shows the most active compound although it was significantly less than that of positive control. The fact that the compounds prepared in this study are chemically unrelated to the current medication suggests that the further work is clearly warranted.

\section{Acknowledgment}

The author A.M.Sridhara thankful to Advinus Therapeutics Pvt.Ltd Bangalore, Chairman, Department of Industrial Chemistry, Kuvempu University for supporting the research.

\section{References}

1. Boland O M, Blackwell C C, Clarke B F and Ewing D J, Diabetes, 1993, 42(2), 336-340.

2. Hamamoto Y, Nagai K, Muto M and Asagami C, Exp Dermatol., 1993, 2(5), 231-235.

3. Yamaguchi M, Kamei K, Koga T, Akima M, Maruyama A, Kuroki T and Ohi N, $J$ Med Chem., 1993, 36(25), 4061-4068.

4. Ono K, Saito T, Sasano H, Moroi R, Sano M and Akimoto T, Xenobiotica, 1979, 9, 227-236.

5. Del Olmo E, Barboza B, Ybarra M I, Lopez-Perez J L, Carron R, Sevilla M A, Boselli C and San Feliciano A, Bioorg Med Chem Lett., 2006, 16(10-15), 2786-2790.

6. Kashima K, Sato N, Sato K, Shimizu H and Mori M, Endocrinol., 1998, 139(8), 3404-3408.

7. Khalil A M, Berghot M A and Gouda M A, Eur J Med Chem., 2009, 44, 4448-4454. 
8. Leenen F H H, Smith D L, Faraks R M, Reeves R A and Marquez-Julio A, Am J Med., 1987, 82, 969-978.

9. Tanaka S, Tanaka M and Akashi A, Stroke, 1989, 20, 1724-1729.

10. Kemp J P, Meltzer E O, Orgel H A, Welch M J, Bucholtz G A, Middleton Jr E, Spector S L, Newton J J and Perhach Jr J L, J Allergy Clin Immunol., 1987, 79, 893-899.

11. Kador P F, Kinoshita J H and Sharpless N E, J Med Chem., 1985, 28(7), 841-849.

12. Mylari B L, Larson E R, Beyer T A, Zembrowski W J, Aldinger C E, Dee M F, Siegel T W and Singleton D H, J Med Chem., 1991, 34, 108-122.

13. Kai H, Matsumoto H, Hattori N, Takase A, Fujiwara T and Sugimoto H, Bioorg Med Chem Lett., 2001, 11(5), 1997-2000.

14. Basappa, Sadashiva M P, Mantelingu K, Swamy S N and Rangappa K S, Bioorg Med Chem., 2003, 11, 4539-4544.

15. Tangallapally R P, Sun D, Rakesh N, Budha N, Lee R E B, Lenaerts A J M, Meibohm B and Lee R E, Bioorg Med Chem Lett., 2007, 17(23), 6638-6642.

16. Srivastava S, Bajpai L K, Batra S, Bhaduri A P, Maikhuri J P, Gupta G and Dhar J D, Bioorg Med Chem., 1999, 7, 2607-2613.

17. Conti P, Dallanoce C, Amici M D, Micheli C D and Klotz K N, Bioorg Med Chem., 1998, 6, 401-419.

18. Saitoh M, Kunitomo J, Kimura E, Hayase Y, Kobayashi H, Uchiyama N, Kawamoto T, Tanaka T, Mol C D, Dougan D R, Textor G S, Snell G P and Itoh F, Bioorg Med Chem., 2009, 17(5), 2017-2029.

19. Chen C J, Song B A, Yang S, Xu G F, Bhadury P S, Jin L H, Hu D Y, Li Q Z, Liu F, Xue W, Lu P and Chen Z, Bioorg Med Chem., 2007, 15, 3981-3989.

20. Iqbal R, Zareef M, Ahmed S, Zaidi J H, Arfan M, Shafique M and Al-Masoudi N A, $J$ Chin Chem Soc., 2006, 53, 689-696.

21. Ram V J and Vlietinck A J, J Heterocycl Chem., 1988, 25, 253-256.

22. Kalsi R, Bhalla T N, Parmar S S and Barthwal J P, Pharmacol., 1989, 39, 103-108.

23. Almasirad A, Vousooghi N, Tabatabai S A, Kebriaeezadeh A and Shafiee A, Acta Chim Slov., 2007, 54, 317-324.

24. Sinay Jr Terry G and Sysko Robert J, U.S. Patent 4904782, 1990.

25. Huisgen R, Angew Chem Int Ed Engl., 1963, 2, 565-598.

26. Liu K C, Shelton B R and Howe R K, J Org Chem., 1980, 45, 3916-3918. 


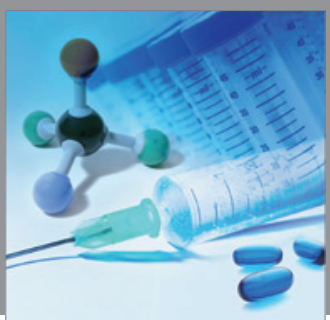

International Journal of

Medicinal Chemistry

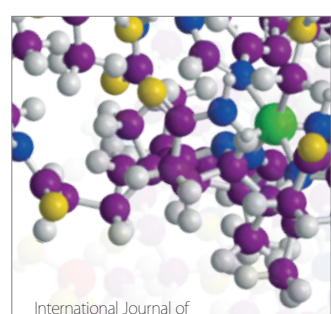

Carbohydrate Chemistry

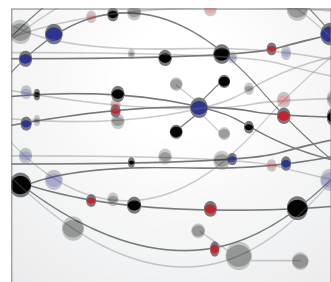

The Scientific World Journal
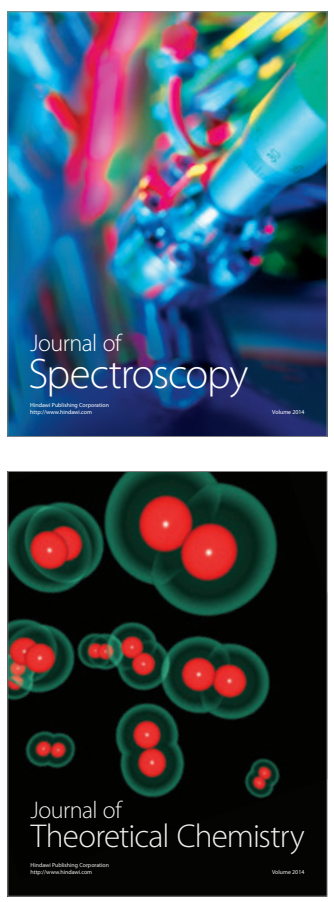
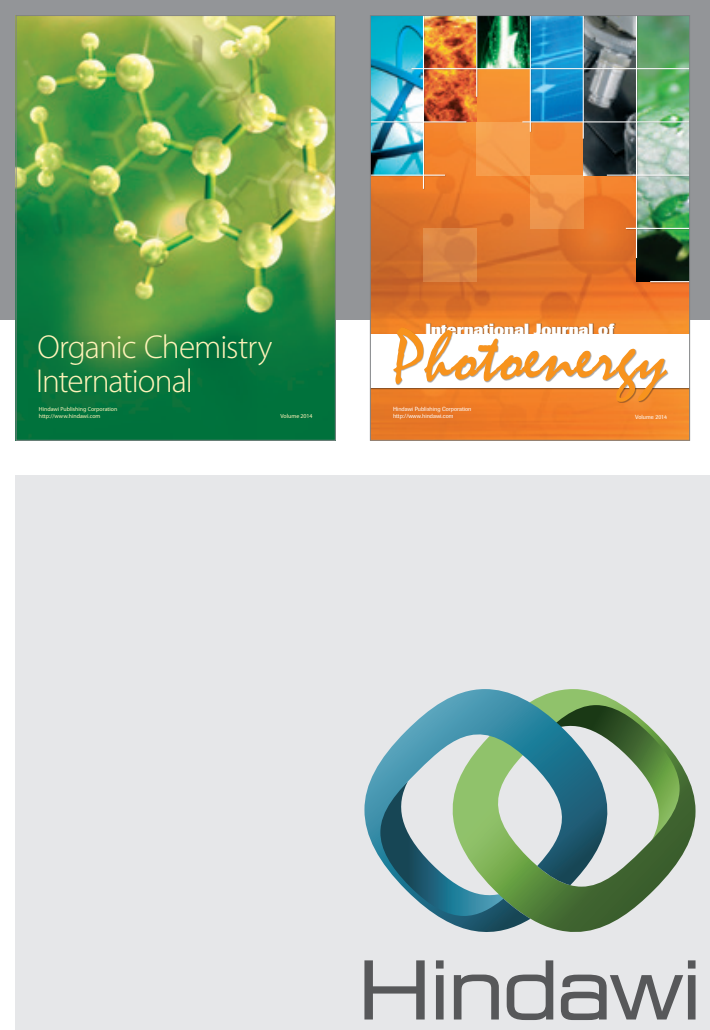

Submit your manuscripts at

http://www.hindawi.com
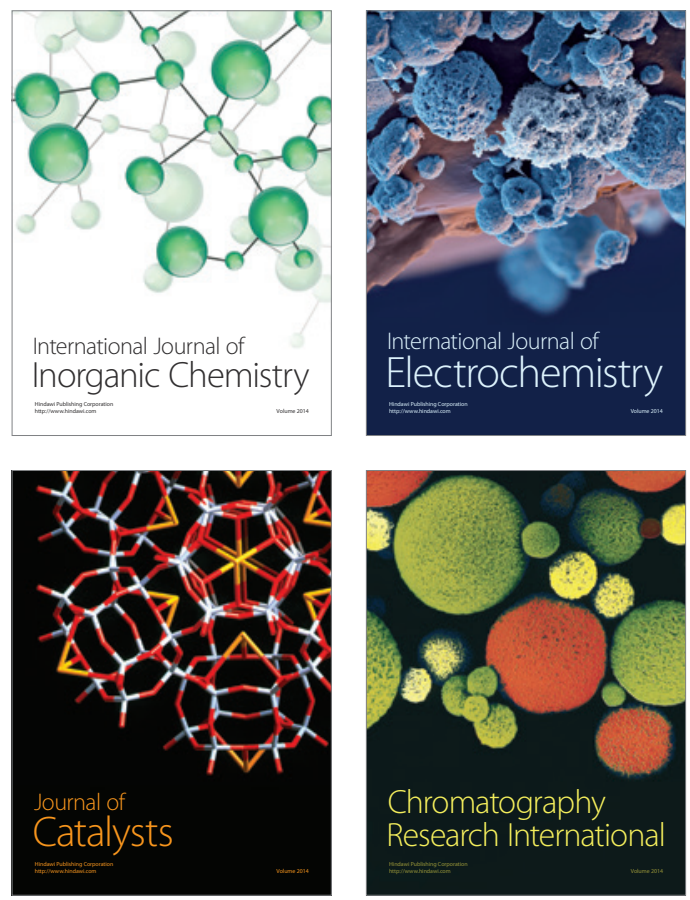
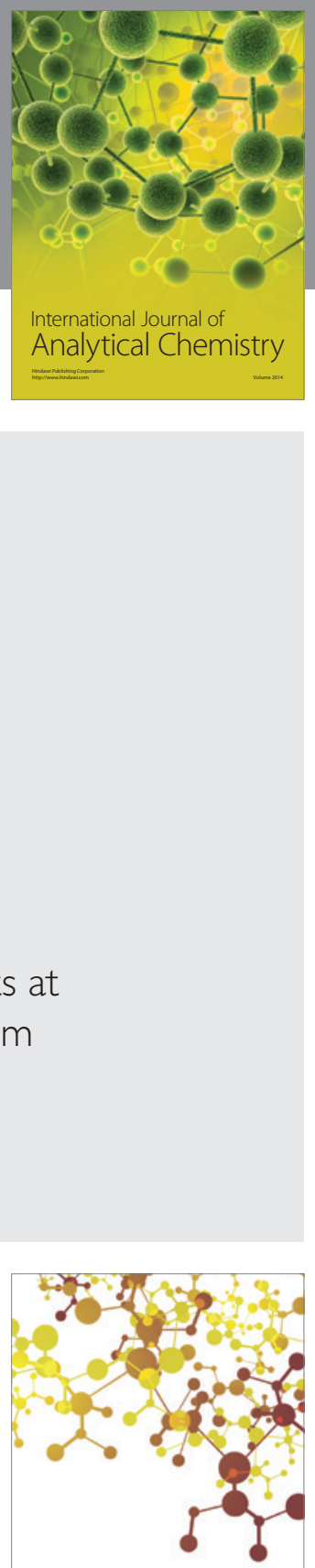

Journal of

Applied Chemistry
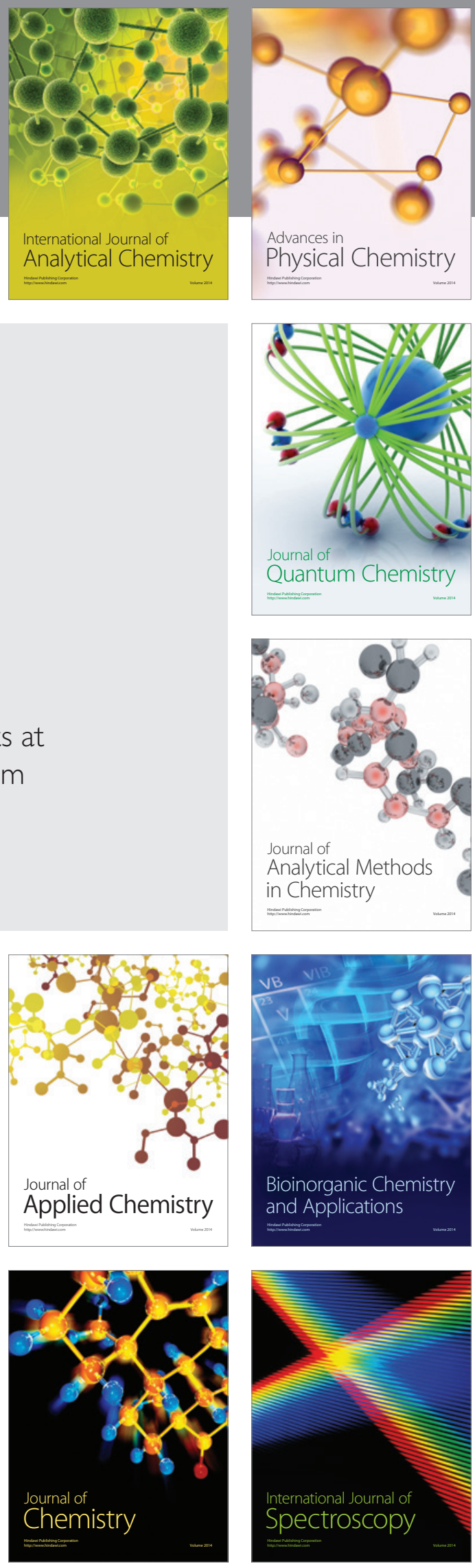